15

\title{
Формирование режима ударного давления в форме бегущей волны в нематических твистовых ячейках
}

\author{
(C) A.B. Захаров ${ }^{1}$, С.В. Пасечник ${ }^{2}$ \\ ${ }^{1}$ Институт проблем машиноведения РАН, \\ Санкт-Петербург, Россия \\ ${ }^{2}$ Московский технологический университет (МИРЭА), \\ Москва, Россия \\ E-mail: alexandre.zakharov@yahoo.ca
}

Поступила в Редакцию 7 октября 2019 г.

В окончательной редакции 7 октября 2019 г.

Принята к публикации 8 октября 2019 г.

\begin{abstract}
Численными методами, в рамках нелинейного обобщения классической теории Эриксена-Лесли, исследован новый нелинейный механизм формирования режима ударного давления на ограничивающие стенки микроразмерной твистовой нематической ячейки (ТНЯ), реализующийся в форме кинкоподобной бегущей волны $\mathscr{P}(z-v t)$, инициируемой как внешним электрическим полем, так и локализованным начальным возмущением поля директора в форме гауссового (нормального) распределения. Изучены механизмы ответственные за формирование бегущей волны ударного давления распространяющегося в ТНЯ от одной ее границы к другой, а также показано, как величина электрического поля и форма локализованного начального возмущения поля директора влияют на сходство бегущей волны с кинкоподобной волной. Результаты исследования динамической релаксации поля директора в ТНЯ также показали, что при температурах, превышающих температуру фазового перехода нематик-смектик $A$ (NA) $T_{\mathrm{NA}}$ на несколько десятков $\mathrm{mK}$, флуктуации параметра порядка зарождающейся смектической фазы подавляют влияние электрического поля и способствуют тому, что плотность азимутальной энергии сцепления демонстрирует сингулярное поведение при $T \rightarrow T_{\mathrm{NA}}$.
\end{abstract}

Ключевые слова: физика жидких кристаллов, гидродинамика анизотропных систем.

DOI: $10.21883 /$ FTT.2020.02.48884.605

\section{1. Введение}

Реальное развитие жидкокристаллических (ЖК) технологий, которые обеспечивают качественное, контрастное и отчетливое изображение на больших ЖК-экранах и панелях, достигается посредством использования супер-твистовой нематической (super twisted nematic) технологии. В основу этой технологии положены твистовые нематические ячейки (ТНЯ) с пикселями, активируемыми электрическим полем $[1,2]$. Невзирая на то, что структурные и оптические свойства твистовых нематических ЖК (НЖК) изучены достаточно подробно [3], еще рано говорить о том, что все механизмы ответственные за динамическую релаксацию как поля директора, так и компонент тензора напряжения ЖК-материала получили достаточное объяснение.

В настоящей статье будет исследован новый механизм, ответственный за формирование нелинейного ударного давления в форме бегущей волны в микроразмерном объеме твистового нематического ЖК (ТНЖК), инициируемого электрическим полем Е. Это явление краткосрочного ударного давления на стенки микроразмерного объема ТНЖК-материала, по-видимому, может быть использовано в конструкциях некоторых микросенсоров и микроактюаторов, в основу которых положены нематические ЖК-материалы [4].
Нематическая твистовая ЖК-ячейка представляет собой микроразмерный объем ЖК-материала, помещенный между двумя параллельными ограничивающими поверхностями, разделенными спейсорами. При этом ориентация директора на верхней поверхности ЖК-ячейки $\hat{\mathbf{n}}^{+}=\hat{\mathbf{n}}_{z=d / 2}$ направлена под прямым углом к ориентации директора на нижней ограничивающей поверхности $\hat{\mathbf{n}}^{-}=\hat{\mathbf{n}}_{z=-d / 2}$, и весь процесс переориентации поля директора $\hat{\mathbf{n}}$ происходит в плоскости, параллельной обеим ограничивающим поверхностям. Здесь $d-$ есть толщина ЖК-ячейки. При отсутствии внешнего поля, под действием только поверхностных сил в такой ТНЯ устанавливается линейное распределение поля директора, характеризующееся азимутальным углом $\psi(z, t)=\frac{\pi}{2} z[5,6]$. Здесь $\psi-$ азимутальный угол, образованный директором $\hat{\mathbf{n}}$ и осью $x$, принадлежащий плоскости ХOY (см. рис. 1). В свою очередь, ось $z$ направлена перпендикулярно ограничивающим поверхностям, а начало отсчета системы координат начинается с середины твистовой ячейки (см. рис. 1). Если одновременно приложить внешнее электрическое поле $\mathbf{E}=E \hat{\mathbf{i}}$, направленное вдоль оси $x$, и придать локальному начальному возмущению поля директора гауссову (нормальную) форму $\psi(z, t=0)=\frac{1}{\sigma} \varphi\left(\left(z-z_{1}\right) / \sigma\right)$, то могут возникнуть несколько режимов релаксации поля 


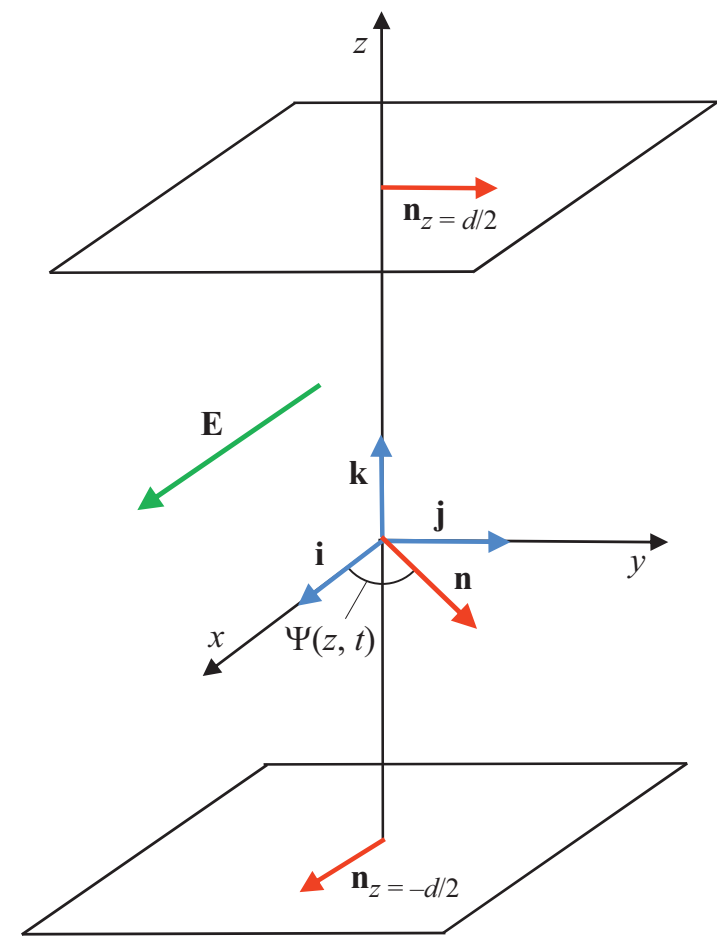

Pис. 1. Геометрия нематической твистовой ячейки.

директора $\hat{\mathbf{n}}(z, t)$ к его равновесному распределению $\hat{\mathbf{n}}_{\mathrm{eq}}$ по объему ТНЯ, направленному вдоль электрического поля Е. Здесь $\varphi\left(\left(z-z_{1}\right) / \sigma\right)$ - гауссова (нормальная) функция распределения, $\sigma$ - ее дисперсия, а $z_{1}-$ положение фокуса гауссового распределения соответственно. До сих пор при исследовании механизмов релаксации поля директора в твистовых НЖК основное внимание было уделено описанию линейных механизмов релаксации $\hat{\mathbf{n}}$ [7], или нелинейных, но не в форме бегущей волны $\psi(z-v t)[6]$.

Новый нелинейный механизм формирования режима ударного давления на ограничивающие стенки микроразмерного объема твистовой ячейки реализующийся в форме кинкоподобной бегущей волны $\mathscr{P}(z-v t)$, инициируемой как внешним электрическим полем, так и локализованным начальным возмущением поля директора в форме гауссового (нормального) распределения, будет описан в рамках нелинейного обобщения классической теории Эриксена-Лесли $[8,9]$, которое учитывает баланс массы, импульсов и угловых моментов действующих на единицу объема ЖК-фазы.

Вышеописанный нелинейный механизм релаксации поля директора в форме кинкоподобной бегущей волны, инициированной как внешним электрическим полем, так и начальным возмущением поля директора, применим при температурах далеких от температур фазового перехода нематик-смектик $A$ (NA) $T_{\mathrm{NA}}$. Вблизи $T_{\mathrm{NA}}$ некоторые материальные коэффициенты, такие как коэффициенты упругости Франка и вращательной вязкости демонстрируют сингулярное поведение при $T \rightarrow T_{\mathrm{NA}}$.
Особенности динамической релаксации поля директора в ТНЯ, при температурах превышающих $T_{\mathrm{NA}}$ на несколько десятков $\mathrm{mK}$ и сопровождающейся формированием смектического домена в нематической фазе, будут также описаны в рамках классической теории ЭриксенаЛесли $[8,9]$.

Статья организована следующим образом: в разделе 2 будет дано описание нового нелинейного механизма релаксации поля директора в ТНЯ в форме бегущей волны, под действием внешнего электрического поля и при температурах соответствующих стабильной нематической фазе, а в разделе 3 будут представлены результаты расчета ряда динамических режимов релаксации как поля директора, так и формирования ударной волны давления на ограничивающие поверхности твистовой ячейки. В разделе 4 будут представлены результаты расчетов ряда динамических режимов возникающих в ТНЯ при температурах близких к температурам фазового перехода нематик-смектик $A$, в то время как в заключительном разделе изложены основные результаты исследования вышеописанных динамических режимов релаксации в твистовых нематических ячейках под действием внешнего электрического поля.

\section{2. Основные гидродинамические уравнения и их решения}

Рассмотрим нематическую твистовую ЖК-ячейку, представляющую собой микроразмерный объем ЖКматериала, при температурах соответствующих стабильной нематической фазе, помещенный между двумя параллельными ограничивающими поверхностями, разделенными спейсорами таким образом, что ориентация директора на верхней поверхности ЖК-ячейки $\hat{\mathbf{n}}^{+}$направлена под прямым углом к ориентации директора на нижней ограничивающей поверхности $\hat{\mathbf{n}}^{-}$. Рассмотрим систему координат отсчитываемую от середины ЖК-ячейки так, что ось $X$ и орт $\hat{\mathbf{i}}$ совпадают с направлением директора на нижней ограничивающей поверхности $\left(\hat{\mathbf{i}} \| \hat{\mathbf{n}}_{\mathrm{z}=-\mathrm{d} / 2}\right)$, в то время как ось $Z$ и орт $\hat{\mathbf{k}}$

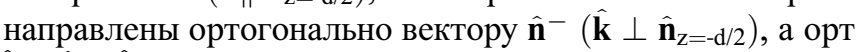
$\hat{\mathbf{j}}=\hat{\mathbf{k}} \times \hat{\mathbf{i}}$. Будем предполагать, что переориентация поля директора $\hat{\mathbf{n}}=\left(n_{\mathrm{x}}, n_{\mathrm{y}}, 0\right)=\cos \psi \hat{\mathbf{i}}+\sin \psi \hat{\mathbf{j}}$ под действием внешнего электрического поля $\mathbf{E}=E \hat{\mathbf{i}}$, направленного вдоль оси $x$, происходит в плоскости ХOY (см. рис. 1.). Поскольку директор $\hat{\mathbf{n}}(z, t)$ остается все время в плоскости $X O Y$, а все пространственные зависимости физических величин вовлеченных в динамический процесс переориентации поля директора сведены к зависимости только от расстояния $z$, отсчитываемого от середины ЖК ячейки, то новый нелинейный механизм формирования режима ударного давления на ограничивающие стенки микроразмерного объема твистовой ячейки реализующийся в форме кинкоподобной бегущей волны $\mathscr{P}(z-v t)$ может быть описан с учетом баланса угловых моментов действующих на единицу объема 
ЖК-фазы как [7]:

$$
\left(\frac{\partial \mathscr{F}_{\text {elast }}}{\partial \psi_{, z}}\right)_{, z}-\frac{\partial \mathscr{F}}{\partial \psi}-\frac{\partial \mathscr{D}}{\partial \psi_{, t}}=0 .
$$

В свою очередь баланс линейных моментов действующих на единицу объема ЖК-фазы может быть записан в виде [7]:

$$
P_{, z}=-\frac{\partial \mathscr{D}}{\partial \psi_{, t}} \psi_{, z} .
$$

Здесь $\mathscr{P}=P-\mathscr{F}-$ произвольное давление в твистовой ЖК-ячейке, $\mathscr{F}=\mathscr{F}_{\text {elast }}-\mathscr{F}_{\text {el }}, \mathscr{F}_{\text {elast }}=\frac{1}{2} K_{2} \psi_{, z}^{2}-$ плотность упругой энергии, приходящейся на единицу объема ЖК-фазы, $\mathscr{F}_{\mathrm{el}}=-\frac{1}{2} \epsilon_{a} \epsilon_{0}(\mathbf{E} \cdot \hat{\mathbf{n}})^{2}-$ плотность электрической энергии, $\mathscr{D}=\frac{1}{2} \gamma_{1} \psi_{, t}^{2}-$ вязкий вклад в полную диссипационную функцию Рэлея, $K_{2}-$ коэффициент упругости Франка, соответствующий твистовой деформации, $\gamma_{1}-$ коэффициент вращательной вязкости, $\epsilon_{a}$ - диэлектрическая анизотропия ЖК-системы, $\epsilon_{0}-$ диэлектрическая проницаемость вакуума, $\psi_{, t}=\frac{\partial \psi}{\partial t}$, $\psi_{, z}=\frac{\partial \psi}{\partial z}$, и $\psi \equiv \psi(z, t)$, а $z$ - расстояние, отсчитанное от центра ЖК-ячейки. В случае жесткого сцепления ЖК-молекул с ограничивающими поверхностями условие баланса угловых моментов на границе раздела ЖК-фаза/твердое тело может быть записано в виде

$$
\psi(z)_{z=-\frac{d}{2}}=0, \quad \psi(z)_{z=\frac{d}{2}}=\frac{\pi}{2} .
$$

Поскольку в нашем случае поле директора $\hat{\mathbf{n}}$ вращается в плоскости $X O Y$, при отсутствии гидродинамического потока $\mathbf{v}=0$, то выражение для произвольного давления $\mathscr{P}$ принимает вид [7]:

$$
\begin{aligned}
\mathscr{P}(z, t)= & \frac{1}{2} K_{2} \psi_{, z}^{2}(z, t)-\frac{1}{2} \epsilon_{a} \epsilon_{0} E^{2} \sin ^{2} \psi(z, t) \\
& -\gamma_{1} \int_{-z / 2}^{z / 2} \psi_{, t}(\chi, t) \psi_{, z}(\chi, t) d \chi .
\end{aligned}
$$

Здесь первые два члена в выражении для произвольного давления соответствуют вкладам упругих и электрических сил, в то время как третий вклад обусловлен вязкими силами. Таким образом, для того чтобы сформировался нелинейный режим ударного давления на ограничивающие поверхности ТНЯ в форме кинкоподобной бегущей волны $\mathscr{P}(z-v t)$, необходимо определить условия, когда возможно формирование нелинейного режима релаксации азимутального угла в форме бегущей волны $\psi(z-v t)$. При этом следует отметить, что поскольку внешнее электрическое поле $\mathbf{E}$ направлено вдоль оси $x$, состояние, соответствующее положению угла $\psi_{z=\frac{d}{2}}(z)=\frac{\pi}{2}$, становится неустойчивым, и кинкоподобный фронт $\psi(z-v t)$ начинает движение от одного края ячейки $\left(z=\frac{d}{2}\right)$ к другому краю $\left(z=-\frac{d}{2}\right)$.

В связи с этим возникает несколько вопросов, например, как быстро фронт бегущей волны будет распространяться в ТНЯ и каково влияние электрического поля и граничных условий на сходство бегущей волны с кинкоподобной волной? На эти и подобные вопросы ответ можно получить с помощью численного решения уравнения баланса угловых моментов действующих на единицу объема ЖК-фазы. В случае плоской геометрии и отсутствия потока $(\mathbf{v}=0)$, уравнение (1) принимает вид

$$
\gamma_{1} \psi_{, t}(z, t)=K_{2} \psi_{, z z}(z, t)+\Delta \sin 2 \psi(z, t),
$$

где $\psi_{, z z}(z, t)=\partial^{2} \psi(z, t) / \partial z^{2}, \Delta=\frac{\epsilon_{0} \epsilon_{a} E^{2}}{2}, \epsilon_{a}=\epsilon_{\|}-\epsilon_{\perp}$, а $\epsilon_{\|}$и $\epsilon_{\perp}-$ коэффициенты диэлектрической проницаемости твистового нематика параллельно и перпендикулярно направлению директора $\hat{\mathbf{n}}$. В уравнении (5) левая часть соответствует вкладу в баланс моментов, обусловленный гидродинамическими силами $\mathbf{T}_{\mathrm{vis}}=-\gamma_{1} \psi_{\text {,t }} \mathbf{\mathbf { k }}$. Это подразумевает отсутствие гидродинамического потока и наличие только вращения поля директора в плоскости $X O Y$.

В случае жесткого сцепления ЖК-молекул с ограничивающими поверхностями условие баланса угловых моментов на границе раздела ЖК-фаза/твердое тело может быть записано в виде уравнения (3), в то время как начальному возмущению поля директора $\psi(z, t=0)$ можно придать форму гауссового (нормального) распределения

$$
\psi(z, t=0)=\frac{1}{\sigma} \varphi\left(\left(z-z_{1}\right) / \sigma\right) .
$$

Начальное условие (6) предполагает, что в момент времени $t=0$ директор сориентирован ортогонально направлению электрического поля $\mathbf{E}=E \hat{\mathbf{i}}$ в форме гауссового распределения со среднеквадратичным отклонением $\sigma$, а затем директору позволено релаксировать к равновесному положению таким образом, что фронт бегущей волны $\psi(z-v t)$ начинает двигаться от одной границы твистовой ячейки $(z=d / 2)$, где директор сориентирован вдоль оси $y$, к другой $(z=-d / 2)$, где директор сориентирован вдоль оси $x$. Отметим, что начальное возмущение в форме гауссового распределения $\varphi\left(\left(z-z_{1}\right) / \sigma\right)$ может быть достигнуто с помощью сфокусированного лазерного импульса.

Определим вначале минимальное значение скорости бегущей волны $v_{m}$ в микроразмерной твистовой ячейке формирующейся под действием внешнего электрического поля $\mathbf{E}$. Для этого подставим выражение для азимутального угла $\psi(z-v t) \sim \exp \left[-E \sqrt{\frac{\epsilon_{0} \epsilon_{a}}{K_{2}}}(z-v t)\right]$ в линеаризованную версию уравнения (5). В результате получим, что минимальная скорость [6] $v_{m}=2 \sqrt{\frac{\epsilon_{0} \epsilon_{a} K_{2}}{\gamma_{1}^{2}}} E$ пропорциональна величине электрического поля $E$, и такая волна может сформироваться в твистовой ячейке толщиной не меньше чем $\kappa=\sqrt{\frac{K_{2}}{\epsilon_{0} \epsilon_{a}}} \frac{1}{E}$. Таким образом, если $E \geq E_{\mathrm{cr}}=\frac{1}{d} \sqrt{\frac{K_{2}}{\epsilon_{0} \epsilon_{a}}}$, то только в этом случае возможно формирование такой бегущей волны, с минимальной 
скоростью $v_{m}$, чтобы она вписалась в твистовую ячейку толщиной $d$.

Для того, чтобы исследовать эту проблему подробнее, перепишем уравнение (5) в безразмерном виде

$$
\psi_{, \tau}(z, \tau)=\psi_{, z z}(z, \tau)+\frac{1}{2} \sin 2 \psi(z, \tau),
$$

где $\tau=\frac{\epsilon_{0} \epsilon_{a} E^{2}}{\gamma_{1}} t-$ безразмерное время, $а$ $=\sqrt{\frac{\epsilon_{0} \epsilon_{a} E^{2}}{K_{2}}} z=z / \kappa-$ безразмерная координата вдоль оси $z$, причем верхняя черта над пространственной переменной $z$ в дальнейшем будет опущена.

Следует отметить, что уравнение (7) является частным случаем более общей проблемы диффузионных уравнений типа [10]:

$$
\psi_{, t}=\psi_{, z z}+f(\psi),
$$

где $f(\psi)$ представляет собой нелинейный функционал, характеризующийся, как минимум двумя положениями равновесия [11]. В конце 30-х годов Колмогоровым и др. было показано [10], что строго локализованное начальное возмущение может асимптотически эволюционировать в форме бегущей волны $\psi(z-v t)$ от одного положения равновесия (неустойчивого) к другому (устойчивому). Это состояние реализуется для широкого класса функционалов $f(\psi)$ при одном условии - чтобы выполнялось $f(0)=0$ и $f^{\prime}(0)=0$. При этом скорость распростронения бегущей волны удовлетворяет неравенствам $2\left[\left(\frac{\partial f(\psi)}{\partial \psi}\right)_{\psi=0}\right]^{1 / 2} \leq v \leq 2\left[\sup _{\psi \in[0,1]}\left(\frac{f(\psi)}{\psi}\right)\right]^{1 / 2}$. В предельном случае эта скорость равна 2 и бегущая волна распространяется в бесконечном интервале $(-\infty,+\infty)$. Но в нашем случае $f(\psi)=\frac{1}{2} \sin 2 \psi(z, \tau)$ и условия $f(0)=0$ и $f^{\prime}(0)=0$ не выполняются, и кроме того мы имеем дело с ограниченным интервалом $[-1 / 2,1 / 2]$, так что бегущая волна $\psi(z-v t)$ сначала должна сформироваться (режим I), а затем распространяться от одного края ячейки $\left(z=\frac{1}{2}\right)$ к другому $\left(z=-\frac{1}{2}\right)$ (режим II) со скоростью $v \geq v_{m}$. Таким образом, наша цель заключается в том, чтобы численными методами, в рамках нелинейного обобщения классической теории Эриксена-Лесли, изучить новый нелинейный, кинкоподобной механизм формирования режима ударного давления $\mathscr{P}(z-v t)$ на ограничивающие стенки микроразмерного объема твистовой ячейки, инициируемый как внешним электрическим полем, так и локализованным начальным возмущением поля директора в форме гауссового (нормального) распределения.

В движущейся системе координат $\xi=z / \kappa-v \tau$ последнее уравнение (7) принимает вид

$$
\psi_{, \tau}(\xi)=v \psi_{, \xi}(\xi)+\psi_{, \xi \xi}(\xi)+\frac{1}{2} \sin 2 \psi(\xi) .
$$

Здесь релаксация поля директора $\hat{\mathbf{n}}$ к его равновесному положению в твистовой ячейке $\hat{\mathbf{n}}_{\mathrm{eq}}$, описывается углом $\psi(\xi)$, который в начальный момент времени претерпевает возмущение в гауссовой форме

$$
\psi(\xi, \tau=0)=\frac{1}{\sigma} \varphi\left(\left(\xi-\xi_{3}\right) / \sigma\right)
$$

где $\varphi\left(\left(\xi-\xi_{3}\right) / \sigma\right)-$ гауссиан со средним $\xi_{3}$ и среднеквадратичным $\sigma$ отклонениями, локализованный вблизи верхней ограничивающей поверхности $\left(\xi_{1}+\xi_{2}\right) / 2<\xi_{3} \leq \xi_{2}$.

В случае жесткого сцепления ЖК-молекул с ограничивающими поверхностями условие баланса угловых моментов на границе раздела ЖК-фаза/твердое тело теперь может быть записано в виде (случай А)

$$
\psi(\xi)_{\xi=\xi_{1}}=0, \quad \psi(\xi)_{\xi=\xi_{2}}=\frac{\pi}{2}
$$

где $\xi_{1}=d /(2 \kappa)-v \tau$ и $\xi_{2}=d /(2 \kappa)-$ соответствуют положениям верхней и нижней ограничивающих поверхностей.

В случае жесткого сцепления ЖК-молекул с верхней и слабого сцепления с нижней ограничивающими поверхностями, при том, что энергия сцепления записывается в форме [12] $W=\frac{1}{2} A \sin ^{2}\left(\psi_{s}-\psi_{0}\right)$, где $A-$ плотность энергии сцепления, а углы $\psi_{s}$ и $\psi_{0}$ отвечают азимутальным углам положения директора на нижней ограничивающей поверхности $\hat{\mathbf{n}}^{-}$и ориентации легкой оси ориентирования $\hat{\mathbf{e}}$, баланс моментов перенесенный на нижнюю поверхность дает граничное условие в виде (случай В)

$$
(\partial \psi(\xi) / \partial \xi)_{\xi=\xi_{1}}=\mathcal{A}, \quad \psi(\xi)_{\xi=\xi_{2}}=\pi / 2
$$

где $\mathcal{A}=\frac{A \kappa}{2 K_{2}} \sin 2 \delta \psi$ и $\delta \psi=\psi_{s}-\psi_{0}$ соответственно.

Процесс релаксации директора к его равновесному положению удобно описать с помощью диссипационной функции $\mathscr{D}$, которая в движущейся системе координат $\xi=z / \kappa-v \tau$ с учетом только твистовых деформаций принимает вид [6] $\mathscr{D}=\psi_{, \tau}^{2}(\xi)$, в то время как выражение для произвольного безразмерного давления $\mathscr{P}$ может быть записано в виде [7]:

$$
\begin{aligned}
\mathscr{P}(\xi) & =\mathscr{P}_{\text {elast }}(\xi)-\mathscr{P}_{\text {el }}(\xi)-\int\left(\partial \mathscr{D} / \partial \psi_{, \tau}\right) \psi_{, \chi}(\chi) d \chi \\
& =-\psi_{, \xi}^{2}(\xi)
\end{aligned}
$$

где $\mathscr{P}_{\text {el }}(\xi)=\frac{1}{2} \sin ^{2} \psi(\xi)$ и $\mathscr{P}_{\text {elast }}(\xi)=-\frac{1}{2} \psi_{, \xi}^{2}(\xi)-$ безразмерные вклады в общее выражение для произвольного давления $\mathscr{P}$ благодаря электрическим и упругим силам. Следует также отметить, что безразмерные $\mathscr{P}$ и размерные $P$ значения давления связаны соотношением $P(\xi)=\left(K_{2} / \kappa^{2}\right) \mathscr{P}(\xi)$ 


\section{3. Результаты вычислений для ряда динамических режимов релаксации поля директора, при температурах, соответствующих стабильной нематической фазе}

Решение уравнения (9) с граничными (11) (случай А) и (12) (случай В) и начальным (10) условиями было получено методом релаксации [13], и при этом критерий сходимости итерационного процесса был выбран в виде $\epsilon=\left|\left(\psi_{(\mathrm{m}+1)}(\xi)-\psi_{(\mathrm{m})}(\xi)\right) / \psi_{(\mathrm{m})}(\xi)\right|$ таким образом, что итерационный процесс продолжался до достижения $\epsilon=10^{-4}$. Здесь $m-$ номер итерации. Результат вычисления эволюции азимутального угла $\psi(\xi)$ в движущейся системе координат $\xi=z / \kappa-v \tau$, под действием электрического поля $E=10 E_{\text {cr }}$, направленного вдоль оси $x$, и для случая жесткого сцепления ЖК-молекул с ограничивающими поверхностями твистовой ЖК-ячейки (случай А), представлен на рис. 2. При этом, начальное возмущение было выбрано в гауссовой форме (10), с локализацией вблизи верхней ограничивающей поверхности $\xi_{3}=4.75$ и дисперсией $\sigma=0.25$. В свою очередь, на рис. 3 представлены результаты расчета эволюции поля директора $\hat{\mathbf{n}}$ к его равновесному положению в твистовой ячейке $\hat{\mathbf{n}}_{\mathrm{eq}}$, описанному углом $\psi(\xi)$ в движущейся системе координат $\xi=z / \kappa-v \tau$, под действием электрического поля $E=10 E_{\text {cr }}$, направленного также вдоль оси $x$, но для случая жесткого сцепления ЖК-молекул с верхней, и слабого сцепления с нижней ограничивающими поверхностями, при том, что энергия сцепления $\mathcal{A}=1.0$. В обоих этих случаях эволюционный процесс, описываемый углом $\psi(\xi)$ в движущейся системе координат $\xi=z / \kappa-v \tau$, из положения характеризуемого углом $\psi(\xi, \tau=0)$ (см. уравнение (10)), в положение характеризующееся углом $\psi_{\text {eq }}(\xi)$, условно можно разделить на две стадии. Первая (стадия I) характеризуется не кинкоподобной эволюцией угла $\psi(\xi)$, вплоть до достижения положений отмеченных на рис. 2 и 3 , номером 1 , в то время как вторая стадия (стадия II) эволюционного процесса, отсчитанная от распределений углов $\psi(\xi)$, помеченных номером 1, и до значений $\psi_{\mathrm{eq}}(\xi)$, помеченных номером 2 , характеризуется кинкоподобной эволюцией угла $\psi(\xi)$. Отметим, что на втором этапе сформировавшеяся кинкоподобная волна характеризуется постоянным профилем $\psi(\xi)$ и безразмерной скоростью пробега $v$. Вычисления показали, что в случае А время, которое система тратит на формирование кинкоподобной волны $\tau_{\mathrm{I}}(A) \sim 3.56$ (режим I), больше чем время пробега кинкоподобной волны $\tau_{\text {II }}(A) \sim 2.38$ (режим II) до достижения равновесной ориентации $\psi_{\mathrm{eq}}(\xi)$. Таким образом, в случае $\mathrm{A}$ общее время релаксации системы состоит из двух вкладов и равно $\tau_{\mathrm{R}}(A)=\tau_{\mathrm{I}}(A)+\tau_{\mathrm{II}}(A) \sim 3.56+2.38=5.94$, в то время как в случае В общее время релаксации равно $\tau_{\mathrm{R}}(B)=\tau_{\mathrm{I}}(B)+\tau_{\mathrm{II}}(B) \sim 4.30+1.58=5.88$ соответственно. В случае А на участке формирования кинкоподобного профиля $\psi(\xi)$ средняя безразмерная скорость равна $v_{\mathrm{I}}(A)=2.5 / 3.56 \sim 0.7$, в то время

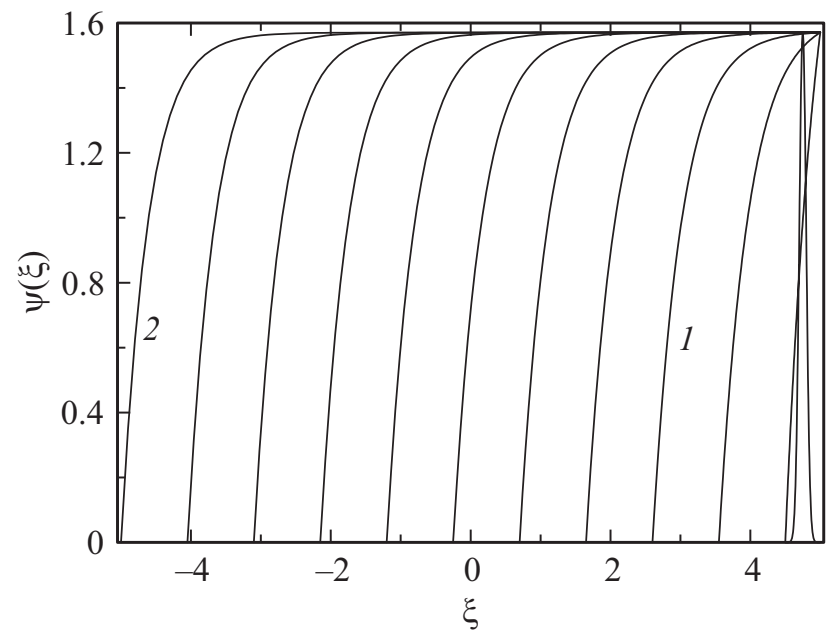

Рис. 2. Эволюция азимутального угла $\psi(\xi)$ vs $\xi=z / \kappa-v \tau$ в микроразмерной ТНЯ под действием электрического поля $E / E_{\mathrm{cr}}=10$, для случая жесткого сцепления ЖК-молекул с ограничивающими поверхностями (случай А). Начальное возмущение выбрано в гауссовой форме $\psi(\xi, \tau=0)=\frac{1}{\sigma} \varphi\left(\left(\xi-\xi_{3}\right) / \sigma\right)$ и локализованно вблизи верхней ограничивающей поверхности $\xi_{3}=4.75$ и $\sigma=0.25$ соответственно.

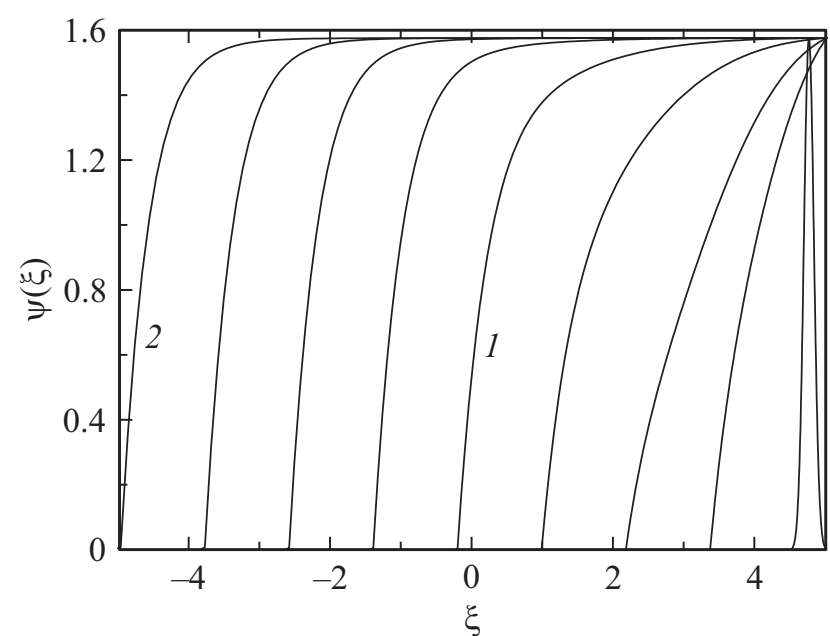

Рис. 3. То же, что на рис. 2, но для случая В, со значением $\mathcal{A}=1$.

как в случае В средняя безразмерная скорость равна $v_{\mathrm{I}}(B)=5.25 / 4.30 \sim 1.22$, что в 1.74 раза выше чем в случае А. В свою очередь, в случае А, на участке, где кинкоподобный профиль $\psi(\xi)$ уже сформировался, средняя безразмерная скорость фронта волны равна $v_{\text {II }}(A)=7.5 / 2.38 \sim 3.15$, в то время как в случае В средняя безразмерная скорость движения фронта кинкоподобной волны равна $v_{\text {II }}(B)=4.75 / 1.58 \sim 3.00$, что в 0.95 раза меньше чем в случае А. Следует отметить, что изменение плотности энергии сцепления ЖК-молекул с нижней ограничивающей поверхностью на один порядок, с $\mathcal{A}=1.0$ до 0.1 , ведет к незначительному уменьшению величины безразмерного времени релакса- 
ции поля директора $\tau_{\mathrm{R}}(B)$ всего на 0.01 безразмерной единицы времени, с 5.88 до 5.87 соответственно.

Отметим, что в случае ЖК-системы, образованной молекулами 4-n'-октил-n-цианобифенила (8ЦБ), при температуре $307 \mathrm{~K}$ значения коэффициента $A$ варьируются между $10^{-5}$ и $10^{-6} \mathrm{~J} / \mathrm{m}^{2}[14,15]$, а значения коэффициента $K_{2} \sim 5.87 \mathrm{pN}$. В случае когда $\Delta \psi<10^{\circ}$ и $d \sim 10 \mu \mathrm{m}$, комбинация $\mathcal{A}=\frac{A \kappa}{2 K_{2}} \sin 2 \delta \psi$ варьируется между значениями 0.1 и 1.0. В свою очередь расчет безразмерной средней скорости $v_{\text {av }}$ вдоль всей толщины твистовой ЖК-ячейки дает следующий результат: в случае $\mathrm{A}, v_{\mathrm{av}}(A)=10 / 5.94 \sim 1.68$, что практически равно значению безразмерной средней скорости $v_{\text {av }}(B)=10 / 5.94 \sim 1.70$, для случая В. Таким образом, можно сделать вывод, что на процесс формирования кинкоподобной бегущей волны $\psi(\xi)$ в микроразмерной твистовой ЖК-ячейке под действием внешнего электрического поля сильное влияние оказывает не только локализация начального возмущения поля директора, но и характер взаимодействия ЖК-молекул с ограничивающими поверхностями. Действительно, положение начального возмущения $\psi(\xi, \tau=0)=\frac{1}{\sigma} \varphi\left(\left(\xi-\xi_{3}\right) / \sigma\right)$ сильно влияет на характер формирования кинкоподобной бегущей волны, и, тем самым, на величину времени релаксации поля директора. Так, если начальное возмущение находится вблизи нижней границы твистовой ячейки $\xi_{3}=-3.7$, то процесс релаксации $\hat{\mathbf{n}}$ к его равновесному положению $\hat{\mathbf{n}}_{\mathrm{eq}}$, в случае В $(\mathcal{A}=1.0)$, реализуется в форме бегущей волны без формирования кинкоподобного профиля $\psi(\xi)$ (см. рис. 4). В этом случае безразмерная величина времени релаксации $\tau_{\mathrm{R}}^{\prime}(B)$ немного возрастает, с $\tau_{\mathrm{R}}(B) \sim 5.88$ до $\tau_{\mathrm{R}}^{\prime}(B) \sim 5.91$.

Результаты расчета средней величины размерной скорости $v_{\mathrm{av}}=d / t_{\mathrm{R}}=\epsilon_{0} \epsilon_{\mathrm{a}} E^{2} d / \gamma_{1} \tau_{\mathrm{R}}$ релаксации поля директора к его равновесному положению, в зависимости от величины электрического поля $E / E_{\mathrm{cr}}$, представлены на рис. 5. Эти вычисления выполнены для случая ЖК-системы образованной молекулами 5ЦБ инкапсулированными в ячейку толщиной $10 \mu \mathrm{m}$. Прежде всего следует отметить, что величина $v_{\text {av }}$ пропорциональна толщине твистовой ЖК-ячейки $d$ и $E^{2}$ и обратно пропорциональна величине коэффициента вращательной вязкости $\gamma_{1}$. Факт слабого влияния величины плотности энергии сцепления $\mathcal{A}$ на среднюю скорость $v_{\mathrm{av}}$ в зависимости от величины поля $E$ отражен в поведении кривых 1 и 2 на рис. 5. Незначительное расхождение в значениях $v_{\text {av }}$, рассчитанных для случаев слабого (случай В) $(\mathcal{A}=1.0)$ (рис. 5, кривая 2) и сильного (случай А)(рис. 5, кривая 1) сцепления ЖК-молекул с ограничивающими поверхностями отмечено лишь при значениях электрического поля $E \sim 9.0 E_{\text {cr. }}$ Кривая 3 (рис. 5) соответствует значениям минимальной скорости $v_{\mathrm{m}}$ распространения бегущей волны в твистовой ЖК-ячейке. Результаты расчета показали, что значения $v_{\mathrm{m}}$ примерно в 3.5 раза меньше обоих значений $v_{\mathrm{av}}(A)$ и $v_{\text {av }}(B)$. Как следует из уравнения (13), формирование бегущей кинкоподобной волны $\psi(\xi)$, движущейся

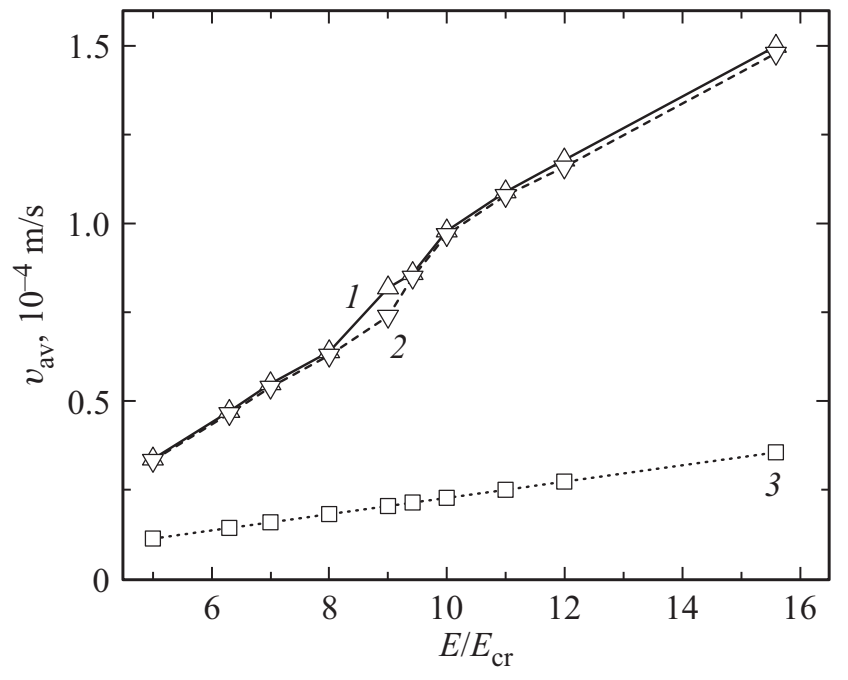

Рис. 4. Зависимость скорости релаксации поля директора $v_{\text {av }}$ от величины электрического поля $E / E_{\text {cr }}$, для случаев жесткого сцепления ЖК-молекул с ограничивающими поверхностями (случай А) (кривая 1), случая жесткого сцепления ЖК-молекул с верхней, и слабого сцепления с нижней ограничивающими поверхностями (случай В) (кривая 2), и минимальной скорости $v_{\mathrm{m}}$ (кривая 3 ), в 10-миллиметровой твистовой ячейке.

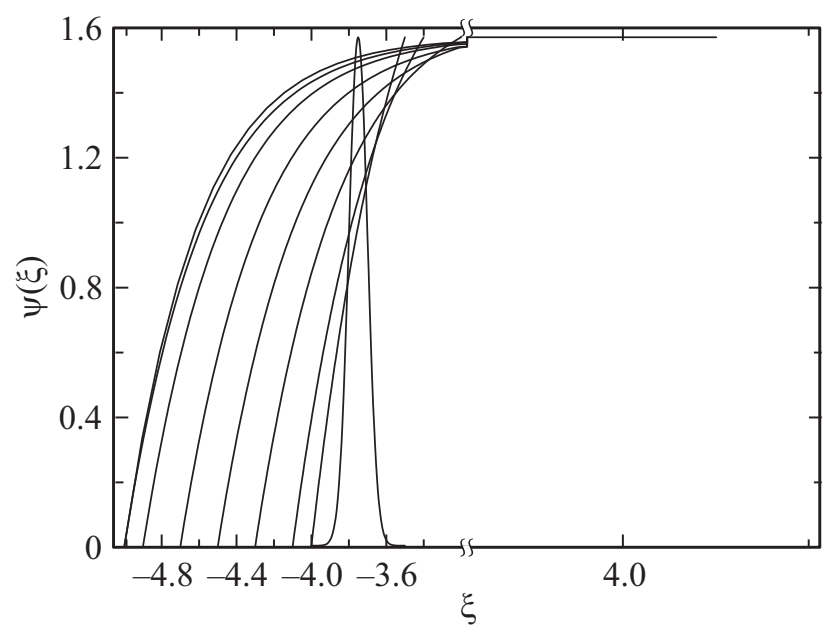

Рис. 5. То же, что на рис. 2, но начальное возмущение локализованно вблизи нижней ограничивающей поверхности $\xi_{3}=-3.7$

поперек твистовой ЖК-ячейки, может инициировать режим бегущего ударного давления $\mathscr{P}(\xi)$ распространяющегося от одной границы ячейки к другой, под действием как внешнего электрического поля $\mathbf{E}$, так и локализованного начального возмущения $\hat{\mathbf{n}}$. Результаты расчетов ударного давления $\mathscr{P}(\xi)$ для случаев жесткого (случай А) и слабого (случай В) граничных сцеплений ЖК-молекул с ограничивающими поверхностями и наличия внешнего поля $E=10 E_{\text {cr }}$ представлены на рис. 6 и 7 соответственно. Показано, что благодаря влиянию как электрического поля $E=10 E_{\mathrm{cr}}$, так и 


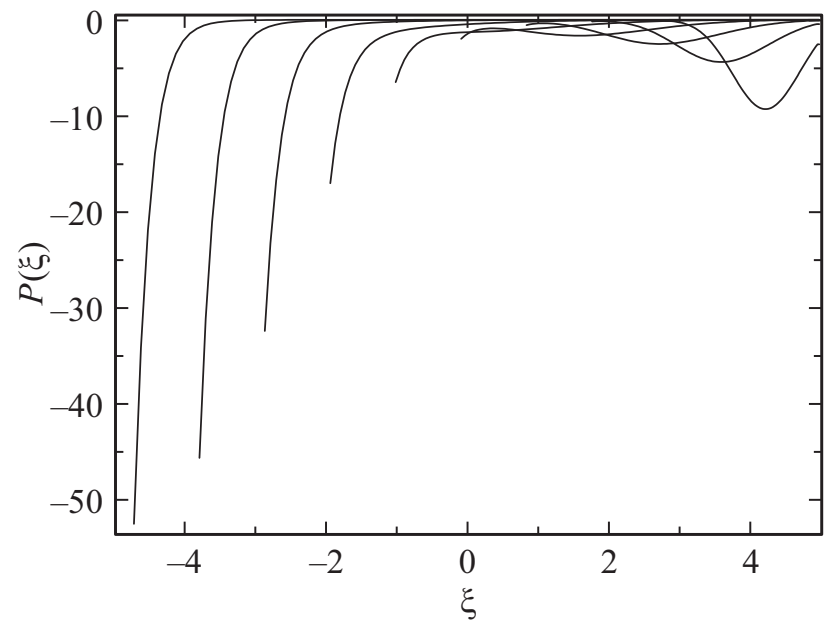

Рис. 6. Эволюция профиля давления $P(\xi)$ vs $\xi=z / \kappa-v \tau$ в микроразмерной ТНЯ под действием электрического поля $E / E_{\mathrm{cr}}=10$, для случая жесткого сцепления ЖК-молекул с ограничивающими поверхностями (случай А). Начальное возмущение выбрано в гауссовой форме $\psi(\xi, \tau=0)=\frac{1}{\sigma} \varphi\left(\left(\xi-\xi_{3}\right) / \sigma\right)$ и локализованно вблизи верхней ограничивающей поверхности $\xi_{3}=4.75$ и $\sigma=0.25$ соответственно.

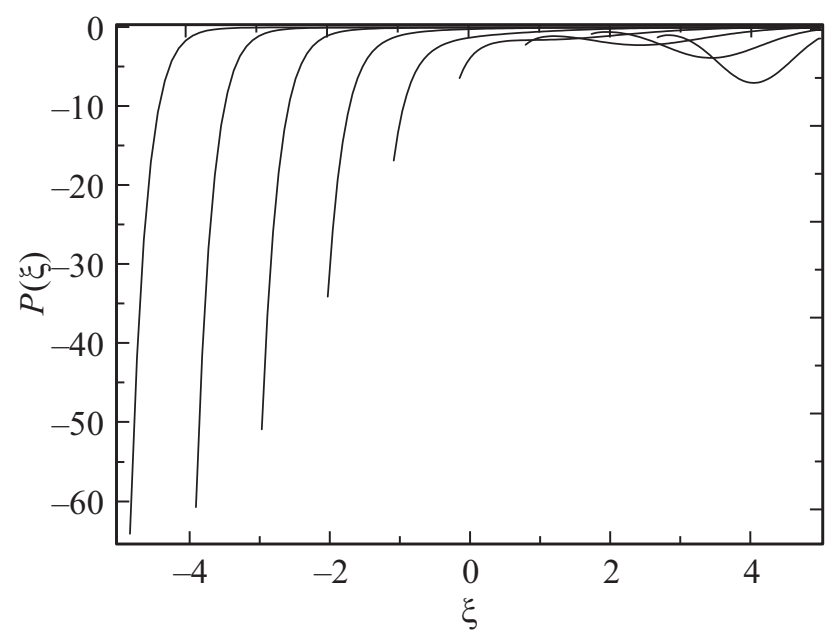

Рис. 7. То же, что на рис. 6, но для случая В, со значением $\mathcal{A}=1$.

начального возмущения поля директора $\hat{\mathbf{n}}$, выбранного в гауссовой форме (см. уравнение (10)), с локализацией вблизи верхней ограничивающей поверхности $\xi_{3}=4.75$ и дисперсией $\sigma=0.25$, на начальном этапе эволюции, абсолютная величина $|\mathscr{P}(\xi)|$ в обоих случаях убывает до нуля (см. рис. 6 и 7), с последующим ростом до $\sim 52$ (случай А) и $\sim 64$ (случай В) безразмерных значений соответственно. Этим безразмерным значениям давления $\mathscr{P}$ соответствуют размерные значения $P(\xi)=\left(K_{2} / \kappa^{2}\right) \mathscr{P}(\xi)$, равные $\sim 5.2 \mathrm{~N} / \mathrm{m}^{2}$ (случай А) и $\sim 6.4 \mathrm{~N} / \mathrm{m}^{2}$ (случай В) соответственно. Расчеты также показали, что профиль ударного давления $\mathscr{P}(\xi)$, расчитанный с учетом упругих, электрических и вязких сил, вначале формируется вблизи верхней ограничивающей поверхности $\xi_{1}=d / 2 \kappa-v \tau$, с последующим движением в сторону нижней ограничивающей поверхности $\xi_{2}=d / 2 \kappa$, которую он достигает спустя время (время релаксации) $\tau_{\mathrm{R}}(\mathrm{I})(\mathrm{I}=\mathrm{A}, \mathrm{B})$. Это как раз то время, которое ЖК-система тратит на переориентацию начального возмущения $\hat{\mathbf{n}}$ под действием внешнего электрического поля $E=10 E_{\text {cr }}$ к его равновесной ориентации $\hat{\mathbf{n}}_{\text {eq }}$. Физически это означает, что в случае формирования бегущей кинкоподобной волны $\psi(z-v t)$ начальное возмущение поля директора вблизи верхней ограничивающей поверхности достигает нижней границы ЖК-ячейки спустя время $t_{\mathrm{R}}(\mathrm{I})(\mathrm{I}=\mathrm{A}, \mathrm{B})$ и оказывает давление $\sim 5.2 \mathrm{~N} / \mathrm{m}^{2}$, в случае $\mathrm{A}$, и $\sim 6.4 \mathrm{~N} / \mathrm{m}^{2}$, в случае В соответственно. Таким образом, эффект слабого сцепления ЖК-молекул с ограничивающими поверхностями ЖК-ячейки приводит к $\sim 20 \%$ росту величины ударного давления на нижнюю ограничивающую поверхность, по сравнению со случаем сильного сцепления. Это в свою очередь показывает, что факт ударного давления на нижнюю ограничивающую поверхность твистовой ЖК-ячейки при вышеописанных условиях спустя время $t_{\mathrm{R}}(\mathrm{I})(\mathrm{I}=\mathrm{A}, \mathrm{B})$ может служить индикатором того, что кинкоподобный фронт бегущей волны уже сформировался и достиг нижней границы ячейки.

\section{4. Аномальная динамика переориентации поля директора вблизи фазового перехода нематик-смектик $\mathrm{A}$}

В случае ЖК-материала такого как 8ЦБ, по мере охлаждения одной из ограничивающих поверхностей, например, нижней, до температур $T$, превышающих $T_{\mathrm{NA}}$ на несколько десятков $\mathrm{mK}$, в пристенной области начинает формироваться смектический домен. Здесь $T_{\mathrm{NA}}-$ температура фазового перехода нематик-смектик A (NA), и в случае 8 ЦБ равная $T_{\mathrm{NA}} \sim 306.5 \mathrm{~K}$ [16]. При этом в нематической фазе в результате флуктуаций зарождающегося смектического порядка ряд материальных параметров, таких как коэффициенты вращательной вязкости $\gamma_{1}$ и упругости Франка $K_{2}$, демонстрируют сингулярное поведение при $T \rightarrow T_{\mathrm{NA}}$. В результате выражения для этих материальных параметров принимают вид [17]:

$$
\begin{aligned}
\gamma_{1}=\gamma_{1}^{\mathrm{G}}+\gamma_{1}^{\mathrm{Cr}} & =\gamma_{1}^{\mathrm{G}}+\frac{k_{B} T}{4} \frac{\pi}{l} \sqrt{\frac{\rho_{m}}{K_{1}}} t^{\nu_{\|}-1}, \\
K_{2}=K_{2}^{\mathrm{G}}+K_{2}^{\mathrm{Cr}} & =K_{2}^{\mathrm{G}}+\frac{k_{B} T}{6} \frac{\pi}{l^{2}} \frac{\xi_{\perp}^{2}}{\xi_{\|}} \\
& =K_{2}^{\mathrm{G}}+\frac{k_{B} T}{6} \frac{\pi}{l^{2}} \frac{\xi_{\perp, 0}^{2}}{\xi_{\|, 0}} t^{-2 v_{\perp}+v_{\|}},
\end{aligned}
$$

где $\gamma_{1}^{\mathrm{G}}$ и $K_{2}^{\mathrm{G}}-$ значения этих материальных параметров при температурах значительно выше температуры $T_{\mathrm{NA}}, k_{B}-$ постоянная Больцмана, $\rho_{m}-$ 
плотность ЖК-фазы, $l$ - расстояние между слоями зарождающейся смектической фазы, $K_{1}$ - коэффициент упругости Франка, соответствующий продольной деформации, $t=T / T_{\mathrm{NA}}-1-$ безразмерная температура, $\xi_{\|}=\xi_{\|, 0} t^{-v_{\|}}$и $\xi_{\perp}=\xi_{\perp, 0} t^{-v_{\perp}}-$ корреляционные длины вдоль и поперек направления поля директора $\hat{\mathbf{n}}$, а $v_{\|}$и $v_{\perp}$ - их критические индексы соответственно.

Следует отметить, что по мере охлаждения нижней ограничивающей поверхности, при $T \rightarrow T_{\mathrm{NA}}$, отношение материальных параметров $K_{2} / \gamma_{1}$ принимает вид

$$
\lim _{t \rightarrow 0} \frac{K_{2}}{\gamma_{1}} \sim \frac{t^{-2 v_{\perp}+v_{\|}}}{t^{v_{\|}-1}}=t^{1-2 v_{\perp}}
$$

В случае ЖК-материала образованного молекулами 8ЦБ значения критических индексов равны $v_{\|} \sim 0.67 \pm 0.02$ и $v_{\perp} \sim 0.55 \pm 0.04$ [14] соответственно, а отношение материальных параметров $\frac{K_{2}}{\gamma_{1}}$, при $t \rightarrow 0$, равно

$$
\lim _{t \rightarrow 0} t^{-(0.1 \pm 0.08)} \rightarrow \infty
$$

Таким образом, в случае жесткого сцепления ЖК-молекул с ограничивающими поверхностями (случай А) уравнение баланса угловых моментов действующих на единицу объема ЖК-фазы, в пределе $T \rightarrow T_{\mathrm{NA}}$, принимает вид (см. уравнение (5))

$$
\psi_{, z z}(z, t)=0
$$

а его решение, с учетом граничного условия (3), когда $\psi(z)_{z=-\frac{d}{2}}=0$, может быть записано в виде

$$
\psi \equiv \psi(z)_{z=-\frac{d}{2}}=0 .
$$

Физически это означает, что по мере того, как $T \rightarrow T_{\mathrm{NA}}$, вблизи нижней ограничивающей поверхности начинает формироваться смектический домен, внутри которого ориентация поля директора не меняется и остается такой же, как на нижней ограничивающей поверхности $z=-\frac{d}{2}$. Фактически, происходит утоньшение области внутри ЖК-ячейки занимаемой нематиком, а нижняя граница сдвигается в сторону верхней ограничивающей поверхности на величину $\xi_{\perp}$.

Этот результат еще важен и потому, что указывает на неограниченный рост времени релаксации поля директора в зарождающемся смектическом домене $t_{R}^{\prime}=\frac{\gamma_{1}^{\mathrm{Cr}}}{K_{2}^{\mathrm{Cr}}} \frac{\xi_{\perp}^{2}}{\bar{E}^{2}} \tau_{R}^{\prime}$. Действительно, по мере охлаждения нижней ограничивающей поверхности до температур превышающих $T_{\mathrm{NA}}$ на несколько десятков $\mathrm{mK}$, время релаксации $\lim _{t \rightarrow 0} t_{R}^{\prime} \sim \lim _{t \rightarrow 0} t^{2 v_{\perp}-1-2 v_{\perp}} \sim \lim _{t \rightarrow 0} t^{-1} \rightarrow \infty$.

Такое поведение релаксационного процесса в смектическом объеме образованном молекулами 8ЦБ, согласуется с оценкой времени релаксации $\lim _{T \rightarrow T_{\mathrm{NA}}} t_{R}^{\prime} \sim \lim _{T \rightarrow T_{\mathrm{NA}}}\left(\frac{T_{\mathrm{NA}}}{T-T_{\mathrm{NA}}}\right)^{0.1}$, полученной методом ЯМР спектроскопии [18].

В случае В, когда молекулы образующие ЖК-фазу жестко сориентированы на верхней и слабо на нижней

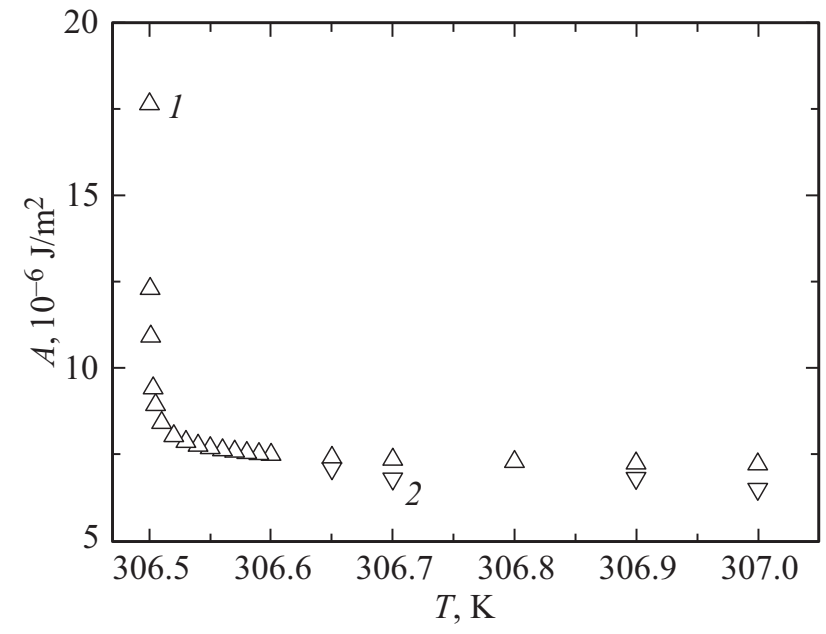

Рис. 8. Зависимость плотности азимутальной энергии сцепления $A$ ЖК-материала, образованного молекулами 8ЦБ, от температуры $T$, рассчитанная с помощью уравнения (19) (помеченные индексом 1), и экспериментальные данные для 8ЦБ, полученные методом динамического рассеяния (помеченные индексом 2) [19].

ограничивающих поверхностях, условие баланса угловых моментов на нижней ограничивающей поверхности, в пределе $T \rightarrow T_{\mathrm{NA}}$, принимает вид

$$
\begin{aligned}
T_{\text {sur }}+T_{\text {anch }} & =\frac{K_{2}}{\kappa} \sin \psi_{s}-\frac{\partial W}{\partial \psi_{s}} \\
& =\frac{K_{2}}{\kappa} \sin \psi_{s}-\frac{A}{2} \sin 2 \Delta \Phi=0 .
\end{aligned}
$$

Здесь $T_{\mathrm{sur}}=\frac{K_{2}}{\kappa} \sin \psi_{s}-$ угловой момент создаваемый упругими силами и стремящийся переориентировать поле директора на нижней ограничивающей поверхности $\hat{\mathbf{n}}_{s}$ вдоль направления электрического поля $\mathbf{E}$, в то время как угловой момент $T_{\text {anch }}=-\frac{\partial W}{\partial \psi_{s}}$ создаваемый силами сцепления ЖК-молекул с нижней ограничивающей поверхностью стремится развернуть вектор $\hat{\mathbf{n}}_{s}$ в сторону $\hat{\mathbf{n}}_{0}, \Delta \Phi=\psi_{s}-\psi_{0}$, а $\kappa=\frac{1}{E} \sqrt{\frac{K_{2}}{\epsilon_{0} \epsilon_{a}}}$ - корреляционная длина электрического поля. Уравнение (18) позволяет оценить плотность азимутальной энергии сцепления $A$ ЖК-молекул с ограничивающей поверхностью

$A=\frac{\pi}{d} \frac{\Phi_{s}}{\Delta \Phi} \bar{E} K_{2}=\frac{\pi}{d} \frac{\Phi_{s}}{\Delta \Phi} \bar{E}\left(K_{2}^{\mathrm{G}}+\frac{k_{B} T}{6} \frac{\pi}{l^{2}} \frac{\xi_{\perp, 0}^{2}}{\xi_{\|, 0}} t^{-2 v_{\perp}+v_{\|}}\right)$,

где $\bar{E}=E / E_{\mathrm{cr}}-$ величина безразмерного электрического поля. На рис. 8 представлены результаты расчета плотности азимутальной энергии сцепления $A$ в зависимости от температуры $T$, для случая ЖК-материала образованного молекулами 8ЦБ, и экспериментальные данные полученные методом динамического рассеяния [19]. В расчетах были использованы значения $K_{2}^{\mathrm{G}} \sim 5.87 \mathrm{pN}$, 
при температуре $307 \mathrm{~K}, \Delta \Phi \sim 10^{\circ}(\sim 0.17) \quad[18]$ и $\Phi_{s}=0.05$. Результаты сравнения указывают на количественное согласие расчетных и экспериментальных данных для величины $A$ в случае ЖК-материала образованного молекулами 8ЦБ.

\section{5. Заключение}

В предлагаемой работе численными методами, в рамках нелинейного обобщения классической теории Эриксена-Лесли, исследован новый нелинейный механизм формирования режима ударного давления на ограничивающие стенки микроразмерного объема твистовой ЖК-ячейки реализующийся в форме кинкоподобной бегушей волны $\mathscr{P}(z-v t)$, инициируемой как внешним электрическим полем, так и локализованным начальным возмущением поля директора в форме гауссового (нормального) распределения. Изучены механизмы, ответственные за формирование бегущей волны ударного давления $\mathscr{P}(z-v t)$, распространяющегося в твистовой нематической ячейке от одной ее границы к другой а также показано, как величина электрического поля и форма локализованного начального возмущения поля директора влияют на сходство бегущей волны с кинкоподобной волной.

Экспериментальное наблюдение релаксационного режима в форме кинкоподобной бегущей волны, повидимому, возможно с помощью поляризационного микроскопа. Поскольку переориентация поля директора имеет место в очень узкой области ЖК-фазы (имеется в виду ширина бегущей волны), под действием внешнего электрического поля $E \geq 10 E_{\mathrm{cr}}$ или $2 \cdot 10^{-4} \mathrm{C} / \mathrm{m}^{2}$ для случая твистовой ЖК-ячейки толщиной $10 \mu \mathrm{m}$, в поляризационном свете могут возникнуть темные бегущие полоски, представляющие собой не что иное как области переориентации поля директора. Учитывая то, что скорость этих полосок $\sim 100 \mu \mathrm{m} / \mathrm{s}$, зарегистрировать данное явление можно наблюдая движение интерференционных полос в новой геометрии (наблюдение в скрещенных поляроидах в плоскости слоя), использованной ранее для регистрации вызванной электрическим полем деформации азимутального поворота директора [2]. Эксперимент такого рода позволит проверить теоретические выводы, представленные в данной статье.

Результаты исследования динамической релаксации поля директора в НТЯ также показали, что при температурах превышающих температуру фазового перехода нематик-смектик A $T_{\mathrm{NA}}$ на несколько десятков $\mathrm{mK}$ флуктуации параметра порядка зарождающейся смектической фазы подавляют влияние электрического поля и способствуют тому, что плотность азимутальной энергии сцепления демонстрирует сингулярное поведение при $T \rightarrow T_{\mathrm{NA}}$.

Исследованные в работе особенности переориентации поля директора в нематических твистовых ячейках под действием внешнего электрического поля необходимо учитывать при создании широкоформатных ЖК-дисплеев и панелей, а также сенсоров и датчиков в основу которых положены ЖК-материалы.

\section{Финансирование работы}

Работа выполнена при финансовой поддержке Министерства образования и науки РФ (гранты $3.11888 .2018 / 11.12$ и $3.9585 .2017 / 8.9)$.

\section{Конфликт интересов}

У авторы заявляют, что у них нет конфликта интересов.

\section{Список литературы}

[1] D.K. Yang, S.T. Wu. Fundamentals of Liquid Crystal Devices. John Wiley and Sons, N.Y. (2006). 387 p.

[2] A.V. Dubtsov, S.V. Pasechnik, D.V. Shmeliova, V.A. Tsvetkov, V.G. Chigrinov. Appl. Phys. Lett. 94, 181910 (2009).

[3] I.C. Khoo. Liquid Crystals: Physical Properties and Nonlinear Optical Phenomena. John Wiley and Sons, N.Y. (1995).

[4] A.P.H.J. Schenning, G.P. Crawford, D.J. Broer. Liquid Crystal Sensors. CRC Press, Taylor and Francis Group, Boca Raton (2018). $164 \mathrm{p}$.

[5] P.G. de Gennes, J. Prost. The Physics of Liquid Crystals. 2nd ed. Oxford University Press, Oxford (1995).

[6] A.V. Zakharov, A.A. Vakulenko. Phys. Rev. E 72, 021712 (2005).

[7] I.W. Stewart. The Static and Dynamic Continuum Theory of Liquid Crystals. Taylor and Francis, London (2004). 345 p.

[8] J.L. Ericksen. Arch. Ration. Mech. Anal. 4, 231 (1960).

[9] F.M. Leslie. Arch. Ration. Mech. Anal. 28, 265 (1968).

[10] W. van Saarloos. Phys. Rev. A 37, 211 (1988).

[11] А.Н. Колмогоров, И.Г. Петровский, Н.С. Пискунов. Бюл. МГУ. Сер. А. Математика и механика 1, 1 (1937).

[12] A. Rapini, M. Papoular. J. Phys. Colloq. (France) 30, C4-541 (1969).

[13] И.С. Березин, Н.Р. Жидков. Методы вычислений. Физматгиз, М. (1964). 464 с.

[14] M. Vilfan, M. Copic. Phys. Rev. E 68, 031704 (2003).

[15] A. Sugimura, K. Matsumoto, O.Y. Zhong-Can, M. Iwamoto. Phys. Rev. E 54, 5217 (1996).

[16] J. Thoen, H. Marynissen, W. Van Dael. Phys. Rev. Lett. 57, 94 (1984).

[17] D. Davidov, C.R. Safynia, M. Kaplan, S.S. Dana, R. Schaetzing, R.J. Birgeneau, J.D. Lister. Phys. Rev. B 19, 1657 (1979).

[18] D. Kamada, K. Okimoto, A. Sugimura, G.R. Luckhurst, B.A. Timimi, H. Zimmermann. Mol. Cryst. Liq. Cryst. 441, 129 (2005).

[19] J.G. Fonseca, Y. Galerne. Phys. Rev. E 61, 1550 (2000).

Редактор Ю.Э. Китаев 